\title{
Telomerase-dependent oncolytic adenovirus for cancer treatment
}

\author{
T-G Huang, MJ Savontaus, K Shinozaki, BV Sauter and SLC Woo \\ Carl C. Icahn Center for Gene Therapy and Molecular Medicine, Mount Sinai School of Medicine, New York, NY, USA
}

\begin{abstract}
Conditionally replicative adenovirus (CRAD) is an attractive anticancer agent as it can selectively replicate in tumor cells. Expression of telomerase reverse transcriptase (TERT) is a unique tumor cell characteristic, being absent in normal postmitotic cells. Thus, we constructed a TERT promoter regulated $C R A D$ for tumor-specific oncolysis by replacing the endogenous adenovirus E1A promoter with that of human TERT (Adv-TERTp-E1A). We showed that its replication was severely attenuated in TERT-negative cells, but that it replicated almost as efficiently as wild-type adenovirus in TERT-positive cells. Accordingly, Adv-TERTp-E1A conferred cytopathicity to TERT-positive, but not TERT-negative, cells. In vivo replication of Adv-TERTp-E1A after local administration into a xenograft model of human hepatocellular
\end{abstract}

carcinoma in nude mice was demonstrated by an increase in adenovirus titers in tumor extracts by several orders of magnitude between $6 \mathrm{~h}$ and 3 days postvector injection. Furthermore, significant inhibition of tumor growth with substantial necrotic tumor areas staining positively for adenovirus was observed with Adv-TERTp-E1A, but not with a control replication-deficient adenovirus. There was also the absence of hepatotoxicity in tumor-bearing animals after intratumoral delivery of the CRAD. The results indicate that the TERT promoter-driven CRAD is capable of tumorselective replication and oncolysis in vitro and in vivo, and can be utilized as an adjuvant treatment agent for cancer. Gene Therapy (2003) 10, 1241-1247. doi:10.1038/ sj.gt.3301987

Keywords: telomerase reverse transcriptase; conditionally replicative adenovirus; cancer gene therapy; hepatocellular carcinoma

\section{Introduction}

Oncolytic viruses, which can replicate selectively in various tumor cell types, provide an attractive strategy for cancer treatment ${ }^{1,2}$ as these viruses replicate only in cancer cells and can result in intratumoral virus spread. Several types of conditionally replicative viruses have already been tested in clinical trials, including conditionally replicative adenovirus (CRAD), herpes simplex virus, vaccinia virus, reovirus and Newcastle disease virus. Two molecular strategies have been developed to target the CRAD selectively to tumor cells. The first strategy involves the deletion of adenovirus genes that are necessary for virus replication in normal cells but not in tumor cells., ${ }^{3,4}$ These include the adenovirus E1A and E1B genes, which are responsible for the inactivation of tumor suppressor $\mathrm{Rb}$ and $\mathrm{p} 53$ genes that are often mutated in cancer cells. This strategy has been effective in animal models, and led to clinical trials combining the application of the E1B/55 kDa-deleted Onyx-015 adenovirus with chemotherapy. ${ }^{5}$ The E1B gene, however, is also involved in the late mRNA export from the nucleus, a property that may ultimately interfere with the efficacy of the Onyx virus as a therapeutic reagent. ${ }^{6,7}$ The second strategy involves the use of tumor- or tissue-specific

Correspondence: Dr SLC Woo, Carl C Icahn Center for Gene Therapy and Molecular Medicine, Mount Sinai School of Medicine, Box 1496, 1425 Madison Avenue, New York, NY, USA

Received 8 October 2002; accepted 23 December 2002 promoters, such as AFP, MUC1, PSA, kallikrein-2 and pS2, to drive adenoviral genes that are essential for replication. ${ }^{8-12}$ This strategy has also been successful in animal models, and the prostate-specific CRADs CN-706 and CV-787 have been tested in clinical trials. ${ }^{11,13}$ This approach, however, is limited to specific tumor types that express the corresponding tumor-specific antigens.

In the present study, we used regulatory elements that are universally active in tumor cells to overcome the limitation imposed by the tissue-restricted expression of various tumor-specific promoters. We chose the telomerase reverse transcriptase (TERT) promoter to drive the adenovirus E1A gene. Telomerase is a DNA polymerase, which directs the synthesis of TTAGGG (telomere) at the ends of chromosomes. As cells continue to divide, telomere shortening occurs and the cells eventually undergo apoptosis. ${ }^{14}$ Telomerase is active in the fetal development and becomes dormant in post-mitotic tissues soon after birth. ${ }^{15}$ High telomerase activity in tumor cells is one of the hallmarks of their 'immortality' and it is active in most human malignancies including lung, liver, gastric, breast, bladder and prostate cancers. ${ }^{14,16-21}$

TERT is the catalytic subunit of telomerase, and it is also a rate-limiting determinant of the enzymatic activity of human telomerase. ${ }^{21}$ It has been shown that TERT is expressed at high levels in malignant tumors and tumor cell lines but is absent in normal postmitotic tissues. ${ }^{20,21}$ In vitro study showed that activation of TERT expression was required for oncogenic transformation of 
telomerase-negative cells. ${ }^{22}$ More importantly, expression of TERT is strictly regulated at the transcriptional level and its promoter has been characterized. The core promoter containing two E boxes and several Sp1 sites is responsible for the trans-activation of TERT. ${ }^{21}$ Furthermore, the TERT promoter has been used to drive Bax as well as thymidine kinase (tk) gene expression for cancer treatment and showed tumor specificity. ${ }^{23,24}$ In the present study, we constructed a universal tumor-specific CRAD, the replication of which is under the control of human TERT promoter, and showed its effectiveness and specificity for tumor treatment in an animal model of human hepatocellular carcinoma.

\section{Results}

\section{Construction of Adv-TERTp-E1A}

A replication-competent adenovirus with tumor specificity was constructed by replacing the endogenous E1A promoter with a human telomerase reverse transcriptase promoter (TERTp) to control the expression of the E1A gene. TERTp, spanning -378 to +76 of the gene and containing the transcriptional start site, is a GC-rich region (GC 78\%). It was amplified with specific primers under conditions as outlined in Materials and methods. The PCR product (TERTp) was then used to replace the endogenous E1A promoter in the pUCE1A plasmid used in our previous study, ${ }^{25}$ and cloned into an adenovirus shuttle vector. The CRAD (Figure 1) was rescued in 293 cells and isolated plaques were propagated in HeLa cells to avoid the formation of wild-type (wt) adenovirus by homologous recombination. No wt adenovirus contaminants were detected in subsequent CRAD preparations as examined by PCR analysis using specific primers covering the adenovirus E1A promoter region.

Specificity of virus replication. To address the issue of replication specificity, replication efficiencies of AdvTERTp-E1A and wt adenovirus were compared in both TERT-positive and -negative cell lines. ${ }^{26-28}$ For every cell line, the relation between multiplicity of infection (MOI) and percentage of target cell infection was firstly determined using an E1A-deleted adenovirus expressing $\beta$-galactosidase. In the subsequent experiments, the MOI that led to $10 \%$ infection of target cells was used in order to be able to observe virus replication in vitro. The cells and supernatants were harvested when cytopathic effects (CPE) became apparent in the infected cells. The virus was released from the cells by freeze-and-thaw

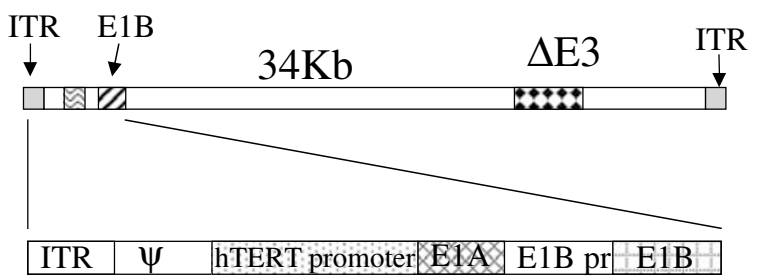

Figure 1 The structure of TERT promoter regulated conditionally replicative adenovirus Adv-TERTp-E1A. TERT promoter replacing endogenous E1A controls the expression of E1A, the E3 including death protein has been removed ( $\triangle E 3)$. ITR, inverted terminal repeat; $\psi$ : packaging signal. cycles and viral titers were determined by plaque assays. As shown in Figure 2, the replication of Adv-TERTp-E1A in TERT-positive cell lines A549, HeLa, Hep3b and HepG2 was within one-log of that of wt adenovirus, whereas its replication in TERT-negative cell lines U2OS and CCD $18 \mathrm{Lu}$ was attenuated by more than 200 -fold when compared to that of wt adenovirus. The results of two independent experiments for each cell line were concordant and showed little variability.

\section{Cell killing effect in vitro}

Two TERT-positive cell lines A549 and Hep3b as well as two TERT-negative cell lines U2OS and CCD 18Lu were infected with Adv-TERTp-E1A, wt adenovirus or a replication-deficient control adenovirus. Cytopathic effects to similar extents were evident in Hep3b and A549 cells after infection with either wt virus or Adv-TERTpE1A (Figure 3a and b). On the contrary, while clear CPE in U2OS and CCD 18Lu cells was evident after wt adenovirus infection, those infected with Adv-TERTpE1A did not display any CPE during the entire time of the experiment (7 and 9 days, respectively) (Figure 3c and d). These results suggested that Adv-TERTp-E1A is capable of TERT-specific oncolysis in vitro.

Viral replication in vivo. To address the in vivo virus replication and tumor killing effectiveness, Hep3b tumors were established by subcutaneous injection of the human hepatocellular carcinoma cells into nude mice. When subcutanesous tumors reached the size of 7-11 $\mathrm{mm}$ in diameter after 2-3 weeks, the CRAD was administrated intratumorally and its replication in the Hep3b tumors was investigated. The injected tumors were explanted at various time points after vector administration and extracts were used to determine infectious viral titers by plaque assay. The total number of infectious viruses detected at $6 \mathrm{~h}$ postinjection was only $9.5 \pm 4.8 \times 10^{3} \mathrm{PFU}$, and it increased dramatically to $14.6 \pm 5.1 \times 10^{7} \mathrm{PFU}$ after $72 \mathrm{~h}$ (Figure $4, P<0.01$ ). The

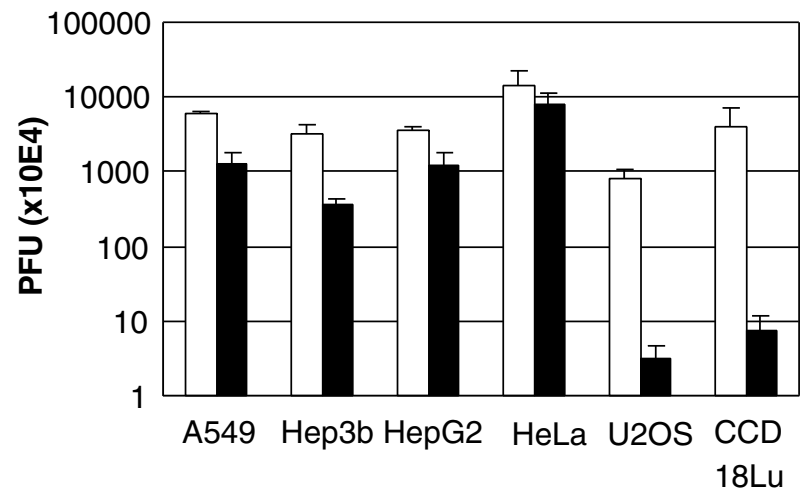

Figure 2 Replication of Adv-TERTp-E1A (solid bars) and Adv-wt (open bars) in human cancer cells in vitro. A549, Hep3b, HepG2 and HeLa cells are telomerase positive, while U2OS and CCD $18 \mathrm{Lu}$ cells are telomerasenegative. The cells were transduced with either Adv-TERTp-E1A or Advwt at an MOI that resulted in $10 \%$ of the cells being infected. The cells were washed and fresh media were added. When CPE became apparent after several days, cells and medium were harvested and lysates prepared from each group. The viral titers and yields from each cell line were determined by plaque assays performed on 293 cells. All experiments have been performed in duplicate. 

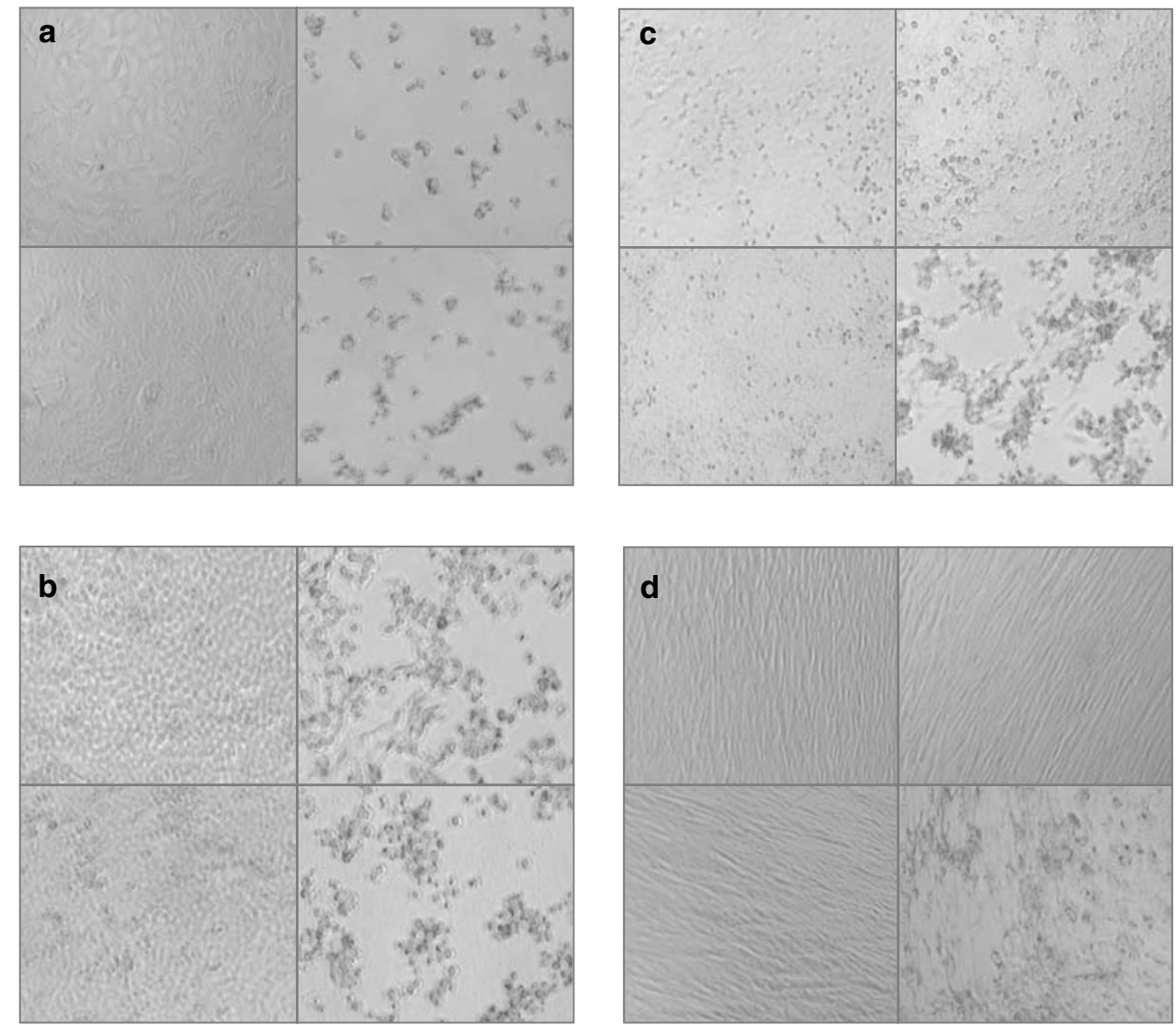

Figure 3 Comparison of cytopathic effect of Adv-TERTp-E1A, wt. adenovirus, replication-deficient control adenovirus (dl-312), and PBS control in TERTpositive cells: (a) Hep3b and (b) A549 as well as in TERT-negative cells; (c) U2OS and (d) CCD 18Lu. Each cell line was infected with either Adv-TERTpE1A (right upper panel), Adv-wt (right lower panel), or dl-312 (left lower panel) at an MOI that resulted in 10\% of the cells being infected (Hep3b and U2OS: MOI of 10; A549 and CCD 18Lu; MOI of 20). PBS control is shown in left upper panel. After infection, the cells were washed and fresh media was added. The cellular morphology was observed daily, and when a clear CPE in the wt adenovirus-infected cells appeared, the experiment was stopped and all panels were photographed: Day 3: Hep3b, Day 6: A549, Day 7: U2OS, Day 9: CCD 18Lu.

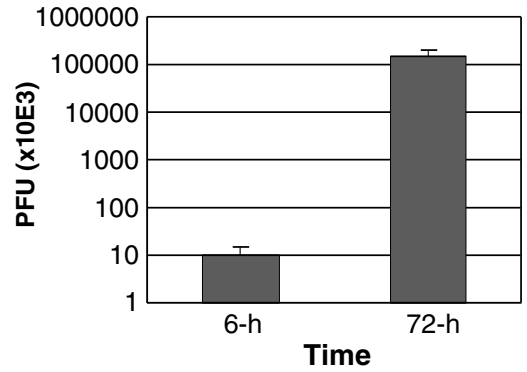

Figure 4 Adv-TERTp-E1A replicated in Hep3b tumor. When Hep3b tumor bearing on nude mice reached $7-11 \mathrm{~mm}$ in diameter, $1 \times 10^{10} \mathrm{vp}$ of Adv-TERTp-E1A was intratumorally injected. Tumors were collected at $6 \mathrm{~h}$ (three tumors) and $72 \mathrm{~h}$ (four tumors) after the virus injection. The tumors were homogenized in 293 cell culture medium and subjected to three cycles of freeze-and-thaw. The supernatant was centrifuged at $10000 \mathrm{~g}$ for $5 \mathrm{~min}$ and viral titers were determined by plaque assays in 293 cells.

greater than 4-log increase in viral titers in tumor extracts over this short period of time provided unambiguous evidence for extensive Adv-TERTp-E1A replication and amplification in the TERT-positive tumors in vivo.

\section{Antitumor effect and viral toxicity in vivo}

In order to determine the efficacy of Adv-TERTp-E1A in tumor treatment, animals with subcutaneous Hep3B tumors of $7-11 \mathrm{~mm}$ in diameter were randomly separated into three treatment groups: Adv-TERTp-E1A $(n=5)$, replication-deficient adenovirus dl-312 $(n=5)$ and vehicle solution control $(n=3)$. Intratumoral administration of a single dose of Adv-TERTp-E1A at $1 \times 10^{10} \mathrm{vp}$ in $100 \mu \mathrm{l}$ significantly inhibited tumor growth as compared with an equivalent dose of dl-312 or with vehicle solution control (Figure 5). This inhibition became apparent at day $8(P<0.05)$, and became magnified over time. The singular Adv-TERTp-E1A treatment, however, did not stop the tumor growth completely. The tumor wet weights at day 28 after the virus injection were $0.7 \pm 0.1,2.2 \pm 0.3$ and $2.5 \pm 0.8 \mathrm{~g}$ for animals treated with Adv-TERTp-E1A, dl-312 and solution control, respectively. The difference between Adv-TERTp-E1A and the two control treatment groups was statistically significant $(P<0.05)$.

The tumors in the three treatment groups were explanted after 28 days and analyzed histologically as well as immunohistochemically. Substantial necrotic areas were presented in Adv-TERTp-E1A-treated tumors. Immunohistochemical staining of adenovirus fibers with Ab-4 showed that only the Adv-TERTp-E1A injected tumors yielded strong positive signals, which superimposed with the necrotic areas (Figure 6). Although there were also necrotic areas in the dl-312injected tumors, they were negative for adenovirus 


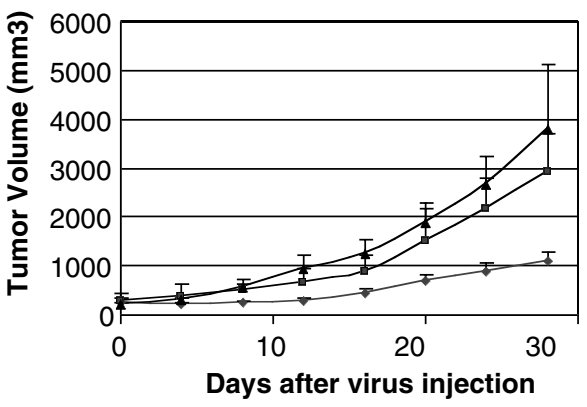

Figure 5 Adv-TERTp-E1A inhibited Hep3b tumor growth in nude mice. When Hep3b tumor reached 7-11 $\mathrm{mm}$ in diameter, the animals were randomly separated into three groups. A measure of $1 \times 10^{10}$ vp of AdvTERTp-E1A (diamond, five mice) or dl-312 (square, five mice) in $50 \mu \mathrm{l}$ of dialysis buffer (10 mM Tris- $\mathrm{HCl}, \mathrm{pH} 7.5,1 \mathrm{mM} \mathrm{MgCl}, 150 \mathrm{mM} \mathrm{NaCl}$ with $10 \%$ glycerol) was injected intratumorally with dialysis buffer (three mice) as a solution control (triangle). The length and width of the tumor were measured every 3 or 4 days. The tumor volume was calculated with the formula (length $\times$ width $\left.{ }^{2}\right) / 2$. All animals were killed at the same time when the length of tumor in control group was bigger than $20 \mathrm{~mm}$.
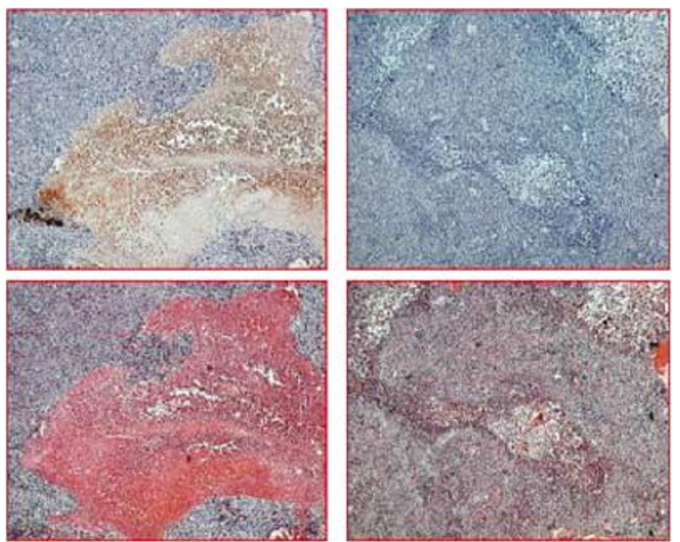

Figure 6 Immunohistochemical staining (IHC) of tumors after virus injection. As indicated in the figure, after animals were killed, the tumors were harvested and fixed. The tissues were stained with antiadenovirus fiber antibody $A b-4$ using $A B C$ method as described in the text. IHC staining: Adv-TERTp-E1A-treated tumors (left upper) showed extensive positive brown adenovirus fiber staining in the necrotic area while dl-312 treated tumors (right upper) revealed only red necrotic tissue $(50 \times)$. The corresponding HEE staining are shown in the lower panel.

staining. The results are suggestive of a causal effect between intratumoral adenovirus replication and tumor necrosis.

Hepatotoxicity is a potentially limiting factor in the use of CRADs as oncolytic agents as adenovirus, and when administered systemically, has a natural tropism for the liver. We therefore examined H\&E sections for inflammatory infiltrates (hepatitis) and measured aspartate aminotransferase (AST) levels in the sera of CRADtreated and dl-312-treated mice after intratumoral vector administration. No liver toxicity was documented in either group (Figure 7).

\section{Discussion}

CRADs are promising new tools for cancer gene therapy because of their potential to specifically replicate and spread in tumor cells, thereby amplifying the tumoricidal effects. In addition to the direct killing by the virus itself, other mechanisms such as augmentation of antitumoral immunity and sensitization of tumors to chemotherapy have also been attributed to the beneficial effects of the CRADs in tumor treatment. Furthermore, CRADs may also be used as a vector to express exogenous therapeutic genes to further potentiate their oncolytic activities. ${ }^{1,2} \mathrm{~A}$ promoter that is active in a wide variety of tumors but not in normal tissues will allow a CRAD to replicate in, and kill, tumor cells irrespective of their tissues of origin. The human TERT promoter is active in most cancer cells and it represents an attractive candidate for the construction of a CRAD that is applicable in the treatment for a wide variety of tumors. In the present study, the human TERT promoter was used to direct the expression of the adenovirus E1A gene, rendering the resulting CRAD to replicate specifically in telomerasepositive tumor cells. We demonstrated that Adv-TERTpE1A replicated in TERT-positive tumor cells in vitro with comparable efficiency to that of wt adenovirus. It also retained its target cell specificity in virus replication, that is, it replicated and killed only TERT-positive Hep3b and A549 cells, but not TERT-negative U2OS and CCD 18Lu cells. A recently published paper reported that a CRAD with TERT promoter-driven E4 failed to control its replication in telomerase-negative cells, ${ }^{29}$ and the authors indicated that E1A gene expression could have activated the E4 gene directly. In our CRAD construct, expression of the E1A gene itself is controlled by the TERT promoter, and specificity for replication in telomerase-positive cells was achieved.

The ability for Adv-TERTp-E1A to replicate intratumorally in vivo was unambiguously demonstrated in a subcutaneous model of human hepatocellular carcinoma in nude mice. Intratumoral virus replication also led to an effective inhibition of tumor growth rate. Hepatotoxicity is obviously a major concern when using replicating adenoviruses. To this end, we demonstrated that intratumoral administration of Adv-TERTp-E1A was not hepatotoxic as evidenced by the lack of inflammatory liver infiltrates (hepatitis) and the absence of liver transaminase elevation in the blood when compared to control adenovirus. The lack of hepatotoxicity has also been reported previously by others using the TERT promoter to direct the expression of Bax or HSV-tk genes in replication-deficient adenovirus vectors. ${ }^{23,24}$

Immunohistochemical studies demonstrated that adenovirus fiber positive areas were present only in AdvTERTp-E1A-injected tumors even after 28 days, but not in those treated with dl-312 and solution controls, and that these areas coincided with the necrotic regions in the tumors. These results suggested that the inhibition of tumor growth was mainly because of necrosis associated with adenovirus replication. In the two control groups, spontaneous necrosis could also be detected in the large tumors, but there was no positive staining for adenovirus. Taken together, the results suggest that the human TERT promoter is a suitable regulatory element to generate tumor-specific CRADs for cancer treatment.

It is evident in this and numerous other studies that the CRADs, despite their powerful oncolytic activities, are not effective as mono-therapeutic agents for cancer. Physical barriers to intratumoral viral spread and generation of effective humoral immune responses 

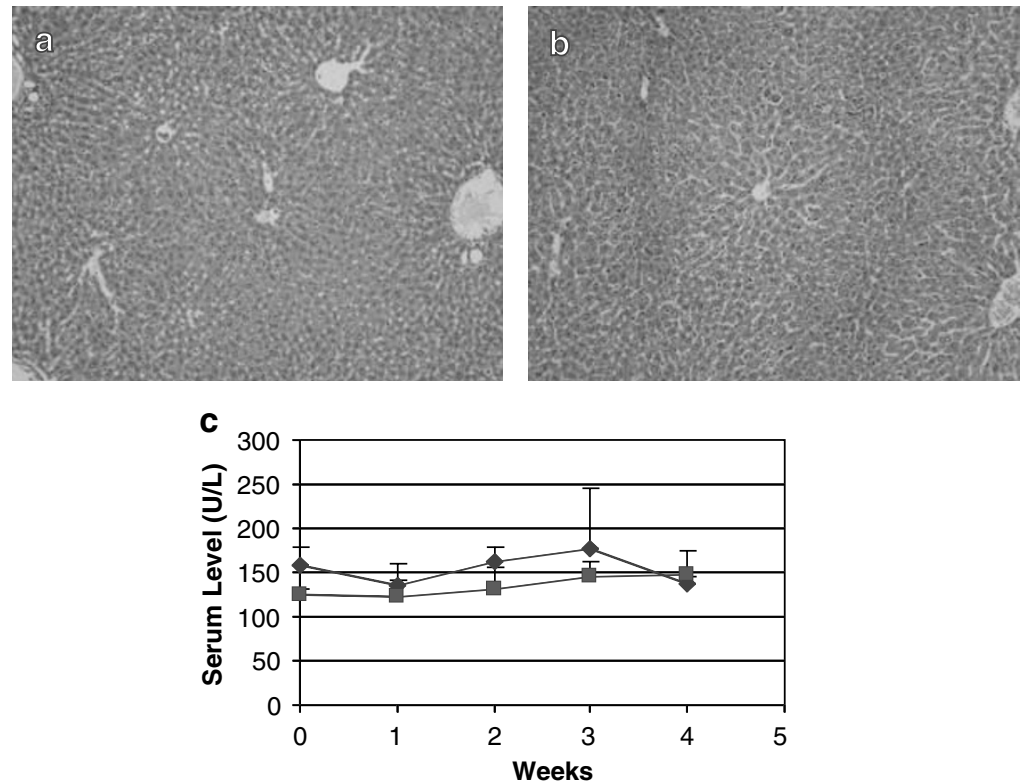

Figure 7 Virus toxicity in vivo: HEE stain of the liver at day 28 after virus injection $(100 \times)$. No inflammatory infiltrates were present in both AdvTERTp-E1A (a), and control vector (dl-312) (b) injected mice. (c) Serum AST levels measured weekly in Adv-TERTp-E1A (diamond) and dl-312 vector (square) injected mice.

against the CRADs in immune-competent hosts will severely limit their effectiveness. This class of agents can nevertheless be used in combination with traditional cancer therapeutic methods, such as chemotherapy and radiation therapy with additive or even synergistic effects. $^{30,31}$ Thus, Onyx-015 is used to treat head and neck, ovarian and colorectal cancers in combination with other chemotherapeutic reagents, 11,32,33 and other CRADs such as CV706, CV787 have been used in combination with radiation or paclitaxel and docetaxel to treat prostate cancer. ${ }^{11,13}$ In this context, we showed in the present study that our telomerase-dependent oncolytic adenovirus was capable of exponential replication in the injected tumors in vivo, thereby significantly reducing the tumor growth rate in a hepatocellular carcinoma model. The telomerase- dependent oncolytic adenovirus may thus be developed as an adjuvant therapeutic agent for a broad range of cancer types in the future.

\section{Materials and methods}

\section{Construction of virus}

For insertion of human TERTp before the E1A transcription start site, a unique AgeI site was created in the adenovirus E1A promoter region of plasmid $\mathrm{pXC1}$ (Microbix Biosystems Inc., Canada). The proximal adenovirus E1A promoter (bp 357-547) was deleted from pXC1 containing adenovirus 5 sequences from bp 22 to $5790^{25}$ and replaced by hTERT promoter as specified below. To generate a functional human TERT promoter, a $454 \mathrm{bp}$ promoter between -378 and +76 was amplified using specific primer pair $5^{\prime}-$ TGGCCCCTCCCTCGGGTTACC-3' (sense) and 5' ${ }^{\prime}$-TAT TACCGGTCGCGGGGGTGGCCGGGGCCAG-3' (antisense) based on published sequence, with an underlined
AgeI tail for cloning. ${ }^{21}$ Human genomic DNA extracted from the whole blood using QIAamp DNA Blood Mini Kit (Qiagen) was used as template, and PFU DNA polymerase (Stratagene, La Jolla, CA, USA) was used for PCR reaction. The first denaturation step was carried out at $97^{\circ} \mathrm{C}$ for $5 \mathrm{~min}$, then at $95^{\circ} \mathrm{C}$ for $45 \mathrm{~s}, 66^{\circ} \mathrm{C}$ for $45 \mathrm{~s}$ and $72^{\circ} \mathrm{C}$ for $45 \mathrm{~s}$. The process was repeated for 30 cycles, followed by an extended period at $72{ }^{\circ} \mathrm{C}$ for $10 \mathrm{~min}$. The PCR product containing the TERTp fragment was cut with AgeI and cloned into the blunted SacII site and AgeI site of pUCE1A to yield pUC-TERTp-E1A. EcoRI-XbaI restriction digestion fragment containing TERTp-controlled E1A was then released from pUC-TERTp-E1A by restriction digestion and cloned into the similarly digested adenovirus shuttle vector pXC1 to yield pXC1-TERTp-E1A. To rescue the virus, $p X C 1-T E R T p-$ E1A and pBHG10 were used to cotransfect 293 cell with the calcium phosphate method, and well-isolated plaques were picked, propagated, molecularly characterized and propagated in HeLa cells. The virus was purified with double cesium gradient ultracentrifugation, and viral particle (VP) was measured by optical absorbance at $260 \mathrm{~nm}$ and plaque-forming units (PFU) were determined by standard agarose-overlay plaque assay on 293 cells. Wild-type adenovirus type 5 (adenoid 75 strain) was obtained from American Type Culture Collection (ATCC, Manassas, VA, USA).

\section{Cell lines}

Human cell lines 293 cell (embryo kidney), A549 (lung cancer), Hep3b and HepG2 (hepatocellular carcinoma), HeLa (cervical cancer), U2OS (osteosarcoma) and CCD $18 \mathrm{Lu}$ (normal lung fibroblast) were purchased from ATCC (Manassas, VA, USA). Cell line 293 was maintained in MEM medium with $10 \%$ fetal bovine serum (FBS) and $100 \mathrm{U}$ penicillin and $100 \mu \mathrm{g} / \mathrm{ml}$ streptomycin; A549 was maintained in Ham's F12 medium with $10 \%$ 
FBS, antibiotics and $2 \mathrm{mM}$ L-glutamine; Hep3b, HepG2, HeLa and CCD 18Lu were maintained in MEM medium with $10 \%$ FBS, $1 \times$ MEM nonessential amino acid (NEAA), $1 \mathrm{~mm}$ sodium pyruvate and $2 \mathrm{mM}$ L-glutamine and antibiotics. U2OS cell was maintained in McCoy 5A with $1.5 \mathrm{mM}$ L-glutamine and 10\% FBS and antibiotics. All liquid reagents for cell culture were purchased from Mediatech Inc. (Herndon, VA, USA).

Virus replication in vitro. Replication of Adv-TERTpE1A in telomerase-positive human cancer cells A549, Hep3b, HepG2 and HeLa cells, and telomerase-negative U2OS and CCD 18Lu cells were studied in vitro with wt adenovirus (Adv-wt) as positive control. The cells were infected with either Adv-TERTp-E1A or Adv-wt or replication-deficient $\mathrm{dl}-312$ at $\mathrm{MOI}$ that resulted in $10 \%$ of the cells being infected. After $1 \mathrm{~h}$ later, the cells were washed and fresh media were added. When CPE in wt control group became apparent after several days, cells and media were harvested combined and lysates prepared from each group by freeze-and-thaw. The viral titer from each cell line was determined by plaque assays performed on 293 cells.

Cell killing study in vitro. As for the replication study mentioned above, human cancer cells A549, Hep3b, HepG2 and HeLa cells, and U2OS as well as CCD 18Lu cells were infected with Adv-TERTp-E1A or Adv-wt as positive control or replication-deficient dl-312 as well as PBS as controls. When CPE in wt control group became apparent after several days, the pictures were taken.

Establishment of Hep3b tumor-bearing model. Female athymic nude mice (10-week old) were used (National Cancer Institute, Frederick, MD, USA). All animal experiments were performed in accordance with the animal guidelines at Mount Sinai School of Medicine, New York, NY. When Hep3b cells grown in $150 \mathrm{~mm}$ dishes were $80 \%$ confluent, the medium was aspirated, and cells were washed two times with prewarmed PBS, and trypsinized. The plates were flapped with hand. When cells detached, the completed medium was added to neutralize the function of trypsin. Cells were pelleted and washed with cold hanks balanced salt solution (HBSS) two times. The cell pellet was resuspended in cold RPMI1640 at a concentration of $2 \times 10^{7}$ cells $/ \mathrm{ml}$ and kept on ice. A total of $4 \times 10^{6}$ cells $(200 \mu \mathrm{l})$ were implanted subcutaneously in the flank of the mice.

\section{Adv-TERTp-E1A replication in Hep3b tumor}

When tumors were $7-11 \mathrm{~mm}$ in diameter, $1 \times 10^{10} \mathrm{vp}$ of Adv-TERTp-E1A in the solution of $10 \mathrm{mM}$ Tris- $\mathrm{HCl}$ ( $\mathrm{pH}$ 7.4) containing $1 \mathrm{mM} \mathrm{MgCl} / 10 \%$ glycerol was injected intratumorally. In total, three tumors were harvested at $6 \mathrm{~h}$ and four tumors harvested at $72 \mathrm{~h}$ after the virus injection. Each whole tumor was homogenized in $1 \mathrm{ml}$ of completed 293 growth medium, and subjected to three cycles of freeze-and-thaw. The supernatant after $10000 \mathrm{~g}$ centrifugation for 5 min was serially diluted and tittered with 293 cell monolayer in six-well plates.

\section{Inhibition of Hep3b tumor growth in nude mice}

When tumors reached $7-11 \mathrm{~min}$ in diameter, $1 \times 10^{10} \mathrm{vp}$ Adv-TERTp-E1A viruses were injected. The tumor size was monitored by calipers every 3 or 4 days. The tumor was measured in its longest dimension (length) and vertical to that (width). The tumor volume was calculated with the formula: volume $=\left(\right.$ length $\times$ width $\left.^{2}\right) / 2$. When the length was larger than $20 \mathrm{~mm}$, the animal was killed. The wet tumor was also weighed. The tumor and liver were fixed in formalin for H\&E stainning.

\section{Immunohistochemical staining}

The tissues obtained as described above in tumor growth study in the nude mice were harvested and fixed with $10 \%$ formalin and embedded with paraffin. The embedded tumors were then cut and dewaxed with serial reduced concentration of ethanol and stained with adenovirus fiber Ab-4 (Clone 4D2, NeoMarkers, Fremont, CA, USA) at the titer of $1: 500$ at $4{ }^{\circ} \mathrm{C}$ overnight, followed by second antibody reaction at 1:300 for $30 \mathrm{~min}$ at room temperature and detected with $\mathrm{ABC}$ complex followed by diaminobenzidine (DAB) and hematoxylin counterstaining.

\section{Statistical analysis}

Unpaired $t$-test was applied to determine the significance of difference.

\section{Acknowledgements}

This work was supported in part by a Department of Defense Grant DAMD17-01-1-0336. We thank Drs. ShuHsia Chen and Simon Hall for helpful and constructive discussions, and Ms Jenny Suriawinata for technical assistance.

\section{References}

1 Kirn D. Replication-selective oncolytic adenoviruses: virotherapy aimed at genetic targets in cancer. Oncogene 2000; 19: 6660-6669.

2 Alemany R, Balague C, Curiel DT. Replicative adenoviruses for cancer therapy. Nat Biotechnol 2000; 18: 723-727.

3 Bischoff JR et al. An adenovirus mutant that replicates selectively in p53-deficient human tumor cells. Science 1996; 274: 373-376.

4 Heise $\mathrm{C}$ et al. An adenovirus E1A mutant that demonstrates potent and selective systemic anti-tumoral efficacy. Nat Med 2000; 6: 1134-1139.

5 Reid Tet al. Intra-arterial administration of a replication-selective adenovirus (d11520) in patients with colorectal carcinoma metastatic to the liver: a phase I trial. Gene Therapy 2001; 8: 1618-1626.

6 Leppard KN, Shenk T. The adenovirus E1B $55 \mathrm{kd}$ protein influences mRNA transport via an intranuclear effect on RNA metabolism. EMBO J 1989; 8: 2329-2336.

7 Shen Yet al. Analyses of single-amino-acid substitution mutants of adenovirus type 5 E1B-55 K protein. J Virol 2001; 75: 4297-4307.

8 Takahashi $\mathrm{M}$ et al. E1B-55K-deleted adenovirus expressing E1A$13 S$ by AFP-enhancer/promoter is capable of highly specific replication in AFP-producing hepatocellular carcinoma and eradication of established tumor. Mol Ther 2002; 5: 627-634.

9 Kurihara T, Brough DE, Kovesdi I, Kufe DW. Selectivity of a replication-competent adenovirus for human breast carcinoma cells expressing the MUC1 antigen. J Clin Invest 2000; 106: 763-771. 
$10 \mathrm{Yu}$ DC et al. The addition of adenovirus type 5 region E3 enables calydon virus 787 to eliminate distant prostate tumor xenografts. Cancer Res 1999; 59: 4200-4203.

$11 \mathrm{Yu}$ DC et al. Antitumor synergy of CV787, a prostate cancerspecific adenovirus, and paclitaxel and docetaxel. Cancer Res 2001; 61: 517-525.

12 Hernandez-Alcoceba R, Pihalja M, Wicha MS, Clarke MF. A novel, conditionally replicative adenovirus for the treatment of breast cancer that allows controlled replication of E1a-deleted adenoviral vectors. Hum Gene Ther 2000; 11: 2009-2024.

13 De weese TL et al. A phase I trial of CV706, a replicationcompetent, PSA selective oncolytic adenovirus, for the treatment of locally recurrent prostate cancer following radiation therapy. Cancer Res 2001; 61: 7464-7472.

14 Shay JW, Zou Y, Hiyama E, Wright WE. Telomerase and cancer. Hum Mol Genet 2001; 10: 677-685.

15 Ulaner GA et al. Telomerase activity in human development is regulated by human telomerase reverse transcriptase (hTERT) transcription and by alternate splicing of hTERT transcripts. Cancer Res 1998; 58: 4168-4172.

16 Hara $\mathrm{H}$ et al. Clinicopathologic significance of telomerase activity and hTERT mRNA expression in non-small cell lung cancer. Lung Cancer 2001; 34: 219-226.

17 Kawakami Y et al. Immuno-histochemical detection of human telomerase reverse transcriptase in human liver tissues. Oncogene 2000; 19: 3888-3893.

18 Rathi A et al. Telomerase RNA expression during progression of gastric cancer. Hum Pathol 1999; 30: 1302-1308.

19 Saito $\mathrm{K}$ et al. Gene expression for suppressors of telomerase activity (telomeric-repeat binding factors) in breast cancer. Jpn J Cancer Res 2002; 93: 253-258.

20 Ito $\mathrm{H}$ et al. Detection of human telomerase reverse transcriptase messenger RNA in voided urine samples as a useful diagnostic tool for bladder cancer. Clin Cancer Res 1998; 4: 2807-2810.

21 Takakura $\mathrm{M}$ et al. Cloning of human telomerase catalytic subunit (hTERT) gene promoter and identification of proximal core promoter sequences essential for transcriptional activation in immortalized and cancer cells. Cancer Res 1999; 59: 551-557.

22 Hahn WC et al. Inhibition of telomerase limits the growth of human cancer cells. Nat Med 1999; 5: 1164-1170.
$23 \mathrm{Gu}$ J, Andreeff M, Roth JA, Fang B. hTERT promoter induces tumor-specific Bax gene expression and cell killing in syngenic mouse tumor model and prevents systemic toxicity. Gene Therapy 2002; 9: 30-37.

24 Majumdar AS et al. The telomerase reverse transcriptase promoter drives efficacious tumor suicide gene therapy while preventing hepatotoxicity encountered with constitutive promoters. Gene Therapy 2001; 8: 568-578.

25 Savontaus MJ, Sauter BV, Huang TG, Woo SL. Transcriptional targeting of conditionally replicating adenovirus to dividing endothelial cells. Gene Therapy 2002; 9: 972-979.

26 Goodwin EC, DiMaio D. Induced senescence in HeLa cervical carcinoma cells containing elevated telomerase activity and extended telomeres. Cell Growth Differ 2001; 12: 525-534.

27 Ogretmen B et al. Molecular mechanisms of ceramide-mediated telomerase inhibition in the A549 human lung adenocarcinoma cell line. J Biol Chem 2001; 276: 32506-32514.

28 Abdul-Ghani R et al. Use of transcriptional regulatory sequences of telomerase (hTER and hTERT) for selective killing of cancer cells. Mol Ther 2000; 2: 539-544.

29 Hernandez-Alcoceba R, Pihalja M, Qian D, Clarke MF. New oncolytic adenoviruses with hypoxia- and estrogen receptorregulated replication. Hum Gene Ther 2002; 13: 1737-1750.

30 Heise $C$ et al. ONYX-015, an E1B gene-attenuated adenovirus, causes tumor-specific cytolysis and antitumoral efficacy that can be augmented by standard chemotherapeutic agents. Nat Med 1997; 3: 639-645.

31 Khuri FR et al. A controlled trial of intratumoral ONYX-015, a selectively replicating adenovirus, in combination with cisplatin and 5-fluorouracil in patients with recurrent head and neck cancer. Nat Med 2000; 6: 879-885.

32 Lamont JP et al. A prospective phase II trial of ONYX-015 adenovirus and chemotherapy in recurrent squamous cell carcinoma of the head and neck (the Baylor experience). Ann Surg Oncol 2000; 7: 588-592.

33 Vasey PA et al. Phase I trial of intraperitoneal injection of the E1B-55-kd-gene-deleted adenovirus ONYX-015 (dl1520) given on days 1 through 5 every 3 weeks in patients with recurrent/ refractory epithelial ovarian cancer. J Clin Oncol 2002; 20: 1562-1569. 